\title{
Discussion on Synchronization of Liver, Spleen and Kidney in Treating Endometriosis from Blood Stasis
}

\author{
Yuanyuan Liu, Xinchun Xiao* \\ Shaanxi University of Traditional Chinese Medicine, Xianyang, Shaanxi, 712000, China.
}

\author{
How to cite this paper: Yuanyuan Liu \\ Xinchun Xiao. (2020) Discussion on \\ Synchronization of Liver, Spleen and \\ Kidney in Treating Endometriosis from \\ Blood Stasitance. International Journal of \\ Clinical and Experimental Medicine \\ Research, 4(4), 242-247. \\ DOI: $10.26855 /$ ijcemr.2020.10.020 \\ Received: October 13, 2020 \\ Accepted: November 6, 2020 \\ Published: November 16, 2020 \\ *Corresponding author: Xinchun \\ Xiao, Shaanxi University of Traditional \\ Chinese Medicine, Xianyang, Shaanxi, \\ 712000, China. \\ Email: xxchsd2003@163.com
}

\begin{abstract}
Endometriosis has a high incidence in women of childbearing age in recent years, and it has received attention because it greatly affects women's physical and mental health. In recent years, Chinese medicine practitioners have clearly confirmed that "stasis" is the main etiology and pathogenesis. However, in the theory of Chinese medicine, the formation of blood stasis is closely related to the main pathogenic factors of liver, spleen and kidney three organ dysfunction. Maintaining vitality, reserving the essence of the kidney, reproducing the main body, and coordinating the liver, spleen, and kidney to harmonize blood, blood, and blood, alleviate and reverse the series of symptoms brought by endometriosis. In terms of the relationship between the formation of endometriosis and irregular menstruation, dysmenorrhea, dysmenorrhea, infertility and other symptoms, it is planned to use the view of liver and spleen and kidney homology in Chinese medicine to explore the relationship between stasis and the incidence of endometriosis To further study the pathogenesis and preventive treatment of endometriosis provides a theoretical basis.
\end{abstract}

\section{Keywords}

Endometriosis, stasis, synchronization of liver, spleen and kidney, treatment based on syndrome differentiation

Endometriosis (endometriosis) is one of the most common diseases in gynecology. It refers to the appearance, growth, infiltration and repeated bleeding of endometrial tissue (glands and stroma) in the endometrium and other parts of the uterus, and then causes pain, infertility, nodules or masses and other symptoms, accounting for about $40 \%$ of the causes of infertility, and $90 \%$ of the patients have pelvic pain [1]. About 176 million women aged 15-49 are currently affected by endometriosis worldwide [2].

Modern medical research has shown that endometriosis is an estrogen-dependent disease [3]. Its treatment is complex, and currently approved hormone therapy for endometriosis can effectively control symptoms, but may have clinically relevant side effects, limiting its long-term use [4]. In 2019, the Chinese Society of Integrated Chinese and Western Medicine Department of Obstetrics and Gynecology issued the latest "Endometriosis Integrated Chinese and Western Medicine diagnosis and treatment guidelines" will belong to the traditional Chinese medicine "blood stasis syndrome" treatment category [5]. Treatment with "removing blood stasis method" can effectively improve the symptoms of discomfort, relieve pain, improve pregnancy rate, and no obvious side effects. Therefore, from the point of view of "blood stasis", combined with clinical and internal heterosis, the etiology and pathological basis of western medicine, further explore the liver, spleen and kidney same way to treat endometri- 
osis pathological mechanism and treatment.

\section{1. "Stasis" is the basic cause and pathogenesis of internal heterosis}

Tang Rongchuan "Blood Syndrome Theory" said: "Since it is blood from the meridian, although clear blood, blood, but also blood stasis". Blood stasis syndrome, as an important syndrome in TCM syndrome differentiation, refers to the performance caused by the stagnation of blood flow in the pulse tube or the stopping of blood accumulation outside the blood overflow pulse [6].

Endometriosis has no special name in traditional Chinese medicine. At present, a large number of doctors have observed that its clinical manifestations and signs are very similar to those of dysmenorrhea, irregular menstruation, infertility and tumor in traditional Chinese medicine. Therefore, referring to ancient books, we can see that ancient doctors often blame the etiology and pathogenesis of similar symptoms of endometriosis as "stasis". "Blood stasis" causes the uterus to flush, qi and blood is not smooth. Then there is no glory, impassable, causing dysmenorrhea; blood stasis stop, Chong Ren damage, cannot become pregnant, so long marriage infertility; blood stasis, collateral obstruction, gradually become a tumor.

Modern studies have also confirmed that patients with endometriosis have slow microcirculation stasis, high blood coagulation state, poor fluidity, strong, sticky, coagulation, aggregation and other characteristics, but after the treatment of blood circulation and blood stasis, microcirculation and blood flow have improved [7]. Therefore, "blood stasis" runs through the development of endometriosis, and "blood stasis" is the most basic pathological basis of endometriosis.

\section{Relationship between blood stasis caused by liver disorders and endometriosis}

\subsection{The liver hides blood and drains}

"Blood is the master of the liver, and evil blood must be attributed to the liver", Li said in the Medical Invention, "Blood Syndrome Theory" said: "The liver is the main blood... Then the liver belongs to wood, wood qi rush and strip reach, do not suppress depression, then the blood vessels are unobstructed”, indicating that liver drainage is very important to the operation of blood. If the liver is lost, it will lead to stagnation of liver qi, stagnation of heat and fire, dampness and heat of liver meridian, hyperactivity of liver yang, internal movement of liver wind and so on, resulting in many gynecological diseases [8]. Drainage function is normal, the air machine will be smooth, and then blood circulation. On the contrary, if the blood flow disorder, will produce blood stasis. Drainage function is normal, the air machine will be smooth, and then blood circulation. On the contrary, if the blood flow disorder, will produce blood stasis [9] Notes. The liver participates in fibrin synthesis and dissolution, and ensures the stability of the coagulation system by removing coagulation factors, fibrinolytic activators, and free heparin. In order to maintain the normal operation of the blood, not to produce blood stasis, internal abnormalities.

\subsection{The liver is connected with Chong and Ren}

"Lingshu Jing” said: “Chong, Ren are all from the cell, on the back, for the sea of meridians". "Chong Xue Hai", "Let the main child", the physiological activities of women such as menstruation, belt, fetus, birth and lactation are all related to rush. If qi and blood deficiency, will lead to blood flow of the degree of unobstructed limited, and then blood stasis block in the Chong, Ren Ermai, slowly will lead to abdominal pain, amenorrhea and other symptoms [10]. The Fu Jueyin Liver Meridian and Ren Mai meet "Qu Gu” [11]. There are many similarities between the liver meridian and Chong Ren two veins. The woman takes the liver as the congenital, the liver blood is full, the two functions of storing blood and dredging each other cooperate and coordinate, the blood sea will overflow on time, rush and let go, and the uterus will leak for a period to maintain the normal function of the woman. Liver loss and catharsis affect Chong Ren, stagnation of liver qi and blood disorder, menstrual blood is not smooth, impassable pain, dysmenorrhea; Qi accumulation blood coagulation, and countercurrent meridian blood mutual cementation, accumulation of abdominal mass, is a tumor; blood stasis, uterine diarrhea dysfunction, so that the two spermatozoa cannot fight, resulting in infertility.

\section{Relationship between Spleen Disorder and Stasis and Endometriosis}

\subsection{Spleen blood, main transport water dampness}

The synopsis of the Golden Chamber says: "The blood of the five viscera and the six entrails depends on the control of temper”. If the function of the spleen is normal, the blood circulation does not overflow the veins and 
cause blood stasis. "Medical Mind Understanding” says: "The spleen is the source of sputum”. A brief summary of the spleen in the water metabolism plays an important role- the transport of water valley fine, scattered its essence to supply the viscera, and prevent the accumulation of excess water in the body. The deficiency of temper is not good, the clear is difficult to rise, the turbid is difficult to fall, the body fluid is abnormal, the water dampness stops inside, and the dampness gathers into phlegm. The solid is because the patient usually eats greasy, continuously injures its spleen function, thus causes the patient to endogenetic phlegm dampness, accumulates the stagnation to stay in the cell palace, causes the patient to have the blood circulation disorder, displays both the qi stagnation and the blood stasis. The symptoms make the blood flow of the patient blocked [12]. Also because of "wet sex trend, easy to attack genitalia", endometriosis is located in the lower coke, blood stasis remains, blood stasis and dampness mutual knot, accumulated for a long time heat, dampness and heat stasis knot, formed the characteristics of internal heterosis cementation difficult to solve, lingering difficult to heal.

\subsection{The spleen is in the middle of the spleen}

Zhu Danxi said: "The spleen has the virtue of Kun Jing, and has the luck of the world, so it can make the heart and lung Yang drop, kidney and liver Yin rise, and become the heaven and earth of the Thai [13]. It shows that the spleen is located in the middle part, and its normal function can make the middle Jiao Qi prosperous, clear Qi is rising, the upper heart and lung are transported, the liver and kidney are issued, and the four sides are irrigated outside. Modern medicine [14-17] Notes, spleen is the largest immune organ in human periphery, which is closely related to cellular immunity, humoral immunity and endocrine metabolism. This function is very similar to the function of spleen in traditional Chinese medicine. Basis of the normal operation of the body's blood-the mutual balance of the anticoagulant and coagulation systems, if this balance is destroyed, it may lead to hemorrhagic disease or thrombus in patients, that is, "blood stasis". The endocrine disorders and metabolic disorders in patients with endometriosis will enhance the platelet activity, and then increase the synthesis and secretion of plasma Pselectin (Ps), Ps through the continuous combination with platelets and endothelial cells, so that the coagulation system of the patient's body can be obtained. To activate, form "stasis" [18-19]. But the experiment [20-23] Confirmation, women's endocrine disorders can lead to a series of symptoms of endometriosis. It shows that spleen dysfunction will affect the operation of qi and blood throughout the body, leading to the formation of blood stasis syndrome. And then lead to irregular menstruation, infertility, tumor and other symptoms.

\section{Relationship between blood stasis caused by renal disorders and endometriosis}

\subsection{Kidney contains essence, main reproduction}

The woman is seven years old, the kidney is full, and the teeth are longer. Two and seven days to, Ren Mai Tong, too Chong pulse Sheng, the month to the present, so there are children... Seven Ren pulse deficiency. Too pulse decline less, day Kui exhausted, tunnel impassable, so bad shape and no son also. "Shenqi Sheng" means that the kidney qi is sufficient, and the two conditions are the growth and development of women, menstruation, birth of children, the prerequisite, basic elements, indicating that kidney and female physiological function is closely related. Kidney contains essence, kidney is the key zang-fu organs of reproduction and development, the essence of the five viscera are attributed to the kidney, essence can blood, blood can produce essence, "essence and blood homology”, kidney yang weakness, then Deficiency of vital qi, lack of warm body, decline of nourishing function, easy to cause cold and dampness, internal obstruction of blood stasis, impassable meridians, discord of qi and blood, and then infertility, dysmenorrhea, tumor and a series of diseases.

\subsection{Kidney system Chong Ren, main birth}

Su asked the theory of strange disease, said: "more than the kidney”. The kidney qi is sufficient, as the place of menstruation and gestation, the palace can get sufficient nourishing and warm, can the qi and blood fill, play its utility. Chong all originated in the cell, by the pulse, through the kidney qi. Therefore, the rise and fall of kidney qi, determine the profit and loss, the palace of Tibetan diarrhea. The kidney essence is sufficient, the pulse can nourish, the blood sea is full, the menstruation can be fixed and down, and the blood is unobstructed. Kidney-TianGui-ChongRen-Cypterian axis interlocking, maintaining the uterine diarrhea, storage overflow. If the kidney is deficient in essence, then Chong, Ren, lost the pulse nourishing, the palace diarrhea dereliction of duty, slow blood, easy to become stasis, Stasis makes blood do not return to menstruation, there will be irregular menstruation, dysmenorrhea, so that eggs cannot be combined and infertility. Such as body and kidney deficiency, Yang Qi inhibition, Yin cold Sheng, Chong Renqi lost in warm, blood for cold coagulation, blood stasis and stag- 
nation for this disease. Blood stasis for Yin evil, blood stasis removal, cell palace unobstructed, if the blood stasis for a long time, Yin essence cannot be transformed, Yang Qi cannot be declared, but also aggravated kidney deficiency. It can be seen that kidney deficiency and blood stasis are causes and consequences, leading to the occurrence of internal disorders, menstrual disorders, dysmenorrhea, and even infertility and other syndromes.

\section{Relationship between liver, spleen and kidney}

Endometriosis is characterized by irregular menstruation, dysmenorrhea, infertility and dysuria. Traditional medicine emphasizes the "holistic view", and man himself is an organic whole. In this whole, meridians, viscera and material functions all cooperate, restrict and influence each other. The pathogenesis of endometriosis is closely related to liver, spleen and kidney. If the liver loses and drains and causes the Qi machine to adjust the function to be abnormal, the liver depression injures the spleen for a long time, the spleen and stomach turns out to be angry and the blood fluid lacks the source, but cannot nourish the whole body, causes the acquired loss of nourishment; the spleen damage is kidney injury, plus the congenital endowment insufficiency, therefore the physique is weak. Long kidney does not hide essence, essence and blood cannot be transformed, cannot be filled with the palace, and gynecological diseases. In addition, the kidney is the birth of the essence of the main viscera and hide, spleen and stomach for the day after tomorrow, the essence of the day after tomorrow water valley scattered, congenital warm to stimulate the day after tomorrow, acquired supplementary cultivation of congenital, congenital and acquired mutual health, mutual promotion, functional coordination. Spleen and liver together for the gas engine hub, the three together regulate the whole body qi and blood operation. Liver, spleen and kidney function can activate blood circulation, nourish essence and store sharp to enhance autoimmune and resist external evil. If their own qi and blood deficiency, the function of the viscera gradually decline, it is easy to lead to the loss of the pulse, and the stagnation of the meridian qi, qi and blood unobstructed, resulting in Chong, Ren, du cell pulse dysfunction, resulting in some miscellaneous diseases [24].

\section{Syndrome Differentiation and Treatment of Internal Disorders Based on "Blood Stasis" and Consistency of Liver, spleen and Kidney}

\subsection{Syndrome Differentiation and Treatment of Internal Disorders Based on "Blood Stasis” and Con- sistency of Liver, spleen and Kidney}

Liver catharsis, Heizuda, evil depression, has the function of regulating emotion, and people in modern society bear all kinds of pressure, the influence of emotion change on liver is very great, heparin depression, or anger injury liver, wood loss, Qi machine is not smooth, blood lag, blood stasis internal obstruction of the uterus, Chong Ren, then developed endometriosis. Qi stagnation and blood stasis syndrome has become the most common syndrome type in modern clinical endometriosis. Qu Qiyun [25] Four inverse San Yuan recipe (Bupleurum 15g,) for relieving depression and soothing liver and regulating qi Radix Paeoniae Alba 20 g, Fructus Aurantii 15 g, Radix Glycyrrhizae $15 \mathrm{~g}$ ) was used to treat dysmenorrhea caused by endometriosis. Wang Hong and other [26] believe that qi stagnation and blood stasis in endometriosis is the most common type, Decoction of Removing Blood Stasis from Diaphragm (Angelica sinensis $10 \mathrm{~g}$, with the function of soothing liver and regulating qi to remove blood stasis Chuanxiong 5 g, 10 g, peony 10 g, of red peony Peach kernel 10 g, 10 g, safflower Pentol 10 g, Annex 10 g, 10 g, of black medicine Fructus Aurantii 10 g, 10 g, Yanhusuo Glycyrrhiza uralensis 15 g) was used in clinic and the control group was set up for observation. The results show that the decoction can effectively reduce the serum CA125, turn EmAB Yin and relieve the pain symptoms of patients with internal heterosis. Hou Jianling [27] The clinical observation found that the drugs of promoting blood circulation and removing blood stasis and regulating qi and relieving pain in Danshen Huoxue decoction are helpful to alleviate the adhesion and reduce the mass of the patients, thus alleviating the pain of the patients and achieving the purpose of relieving the symptoms quickly.

\subsection{Invigorating spleen and removing blood stasis}

"The origin of the disease" cloud: "all phlegm, this by blood congestion, drinking water accumulation and not dissipate, so phlegm also". This article shows that phlegm turbid due to blood congestion, drinking water accumulation, blood stasis for a long time, stasis Qi stagnation, Sanjiao gasification is not smooth, then wet into phlegm. Whether it is dampness and heat stasis knot or phlegm and blood stasis caused by irregular menstruation, dysmenorrhea or infertility, in the final analysis, but the usual food paste thick taste, or liver Wangke spleen, spleen deficiency transport loss, phlegm turbid endogenetic, and blood from the meridian, phlegm and blood stasis mutual knot, rushed or not solid. Fu Shuxiu [28] etc. Composing spleen and removing blood stasis formula g, 
Codonopsis 15 Cloud Poria cocos 15g, 10g, of Atractylodes macrocephala Astragalus g,15 Peach kernel 10 g, 10 g, pastry $15 \mathrm{~g}$, of red peony Guizhi 6g, 30g, of soap Curcuma g.10 Anal pain obvious plus $6 \mathrm{~g}$, of wood incense Black medicine 6 g; 6 g, of Evodia rutaecarpa plus Gun 6 g; Add 6 g, to the yellow 10 g. Scutellaria baicalensis Clinical observation of 46 patients with endometriosis. It was found that tonifying spleen can effectively treat a series of symptoms caused by endometriosis. Yue Xiaoqiang [29] etc. add and subtract Yiqi decoction and Xiaoxiao Powder (Radix Glycyrrhizae 6 g, Chen Pei 12 g, Use cases 15 g, Bupleurum 9 g, Rhizoma cimicifugae 6 g, White peony 18 g, Salvia miltiorrhiza 18 g, Chicken blood vine 30 g, Pollen Typhae 9 g, Wulingzhi 9 g, Biejia 12 g, Chicken nugget 18 g, Codonopsis pilosula 30 g, Roasted Astragalus 30 g, Atractylodes macrocephala 30 g) to invigorate qi and spleen, Promoting blood circulation and removing blood stasis in the treatment of symptoms brought by endometriosis has a better effect.

\subsection{Treatment of Endometriosis by Invigorating Kidney and Removing Blood stasis}

Kidney deficiency is a disease, whether kidney yin deficiency or kidney yang deficiency will have pathological changes caused by deficiency. Choroid stasis, blood flow is not smooth, it hinders the kidney yin, kidney yang metaplasia. Wang Wenjuan etc. [30] Using the method of tonifying kidney and removing blood stasis to divide the patients with endometriosis according to the stage before and after ovulation, Preparation before ovulation (Rehmannia glutinosa 20 g, Cuscuta 20 g, Angelica sinensis 15 g, Salvia miltiorrhiza 15 g, Wulingzhi 15 g, Radix Paeoniae Alba 15 g, Zedoary 12 g, chicken blood rattan 12 g, fragrant 12 g, Sichuan Achyranthes bidentata 12 g; Poria cocos 10 g, Yanhusuo $10 \mathrm{~g}$, Guizhi $10 \mathrm{~g}$ ) activating blood stasis, tonifying kidney and nourishing blood. It can promote the transformation of yin and yang in the kidney 25 g, after ovulation Cuscuta chinensis 20 g, Chicken blood vine 15 g, Epimedium 15 g, Ai Ye 15 g, Yanhusuo 15 g, Guizhi 15 fried white Paeonia lactiflora 15 g, Glycyrrhiza uralensis $10 \mathrm{~g}$ ) with regulating menstruation and relieving pain and tonifying kidney and warming meridian as the main treatment principle, the clinical effect is remarkable and effective. Modern pharmacology has confirmed that tonifying the kidney and removing blood stasis can effectively regulate the endocrine of patients and increase the immune function, which has a better effect on the symptoms caused by endometriosis. Han Liyan etc. [31] To remove turbidity, detoxify kidney, remove blood stasis, draw up the prescription of tonifying kidney and removing Rehmannia glutinosa 20 g, Dodder 20 g, Chinses angelica 15 g, Salvia miltiorrhiza Bge 15 g, Wulingzhi 15 g, Red Peony15 g, Rhizoma Curcumae 12 g, Caulis Spatholobus 12 g, Rhizoma Cyperi 12 g, Sichuan Ach knee $12 \mathrm{~g}$; Poria cocos $10 \mathrm{~g}$, Yancho $10 \mathrm{~g}$, Guizhi $10 \mathrm{~g}$ was used in endometriosis model rats. At the molecular level, it is verified that the prescription of removing turbid and detoxifying kidney and removing blood stasis may promote blood operation, to improve the condition of ischemia and hypoxia, enhancing HIF-1 $\alpha$ expression, to treat the uterus the efficacy of the aim of endometriosis.

\section{Conclusion}

For endometriosis, it is mainly due to the imbalance of liver, spleen and kidney, stagnation of qi and blood, and unobstructed meridian qi, which leads to local lesions. It is found that the basic methods of treating endometriosis, whether based on clinical practice or laboratory exploration, cannot be separated from the treatment of endometriosis.

\section{References}

[1] Kodaman, P. H. (2015). Current strategies for endometriosis man-agement [J]. Obstet Gynecol Clin North Am, 2015, 42(1): 87-101.

[2] Rocha, A. L., Reis, F. M., Taylor, R. N. (2013). Angiogenesis and endometriosis [J]. Obstet Gynecol Int, 2013: 859619.

[3] Kennedy, S., Bergqvist, A., Chapron, C., et al. (2005). ESHRE guide-line for the diagnosis and treatment of endometriosis [J]. Hum Reproduction, 2005, 20(10): 2698-2704.

[4] Safety of Dienogest and Other Hormonal Treatments for Endometriosis in Real-World Clinical Practice (VIPOS): A Large Noninterventional Study.

[5] Yu Chaoqin, Duan Hua, Xu Hong, et al. (2019). Guidelines for the diagnosis and treatment of endometriosis [J]. Chinese Journal of Integrated Chinese and Western Medicine, 2019(10): 1169-1176.

[6] Ji Shaoliang, Cheng Zhaozhi. (2009). TCM Diagnostics [M]. Beijing: People’s Health Publishing House, $2009: 126$.

[7] Liu Jinxing, Mao Furong, Zhang Yingchun. (1994). Clinical Study on Treating Endometriosis with Removing Blood Stasis and Eliminating Phlegm and Soft Jian Powder [J]. Chinese Journal of Integrated Chinese and Western Medicine, 1994, 14(6): 337-339. 
[8] Yang Xianhai, Yuan Fahui, Chai Jinhua, et al. (2013). Li Zhiyuan’s Academic Thought on the Treatment of Gynecology with Liver [J]. Modern Distance Education in Chinese Traditional Chinese Medicine, 2013, 011(003): 96-98.

[9] Fu Zhengwen. (2017). Changes of blood clotting function index in patients with hepatitis B, liver cirrhosis and liver failure [J]. Thrombosis and Hemostasis, 2017, 23(4): 609-611.

[10] Shen Chunhui. (2014). Efficacy of laparoscopic combined repair of diverticulum of uterine incision after cesarean section in 48 cases\% Effect Observation on Hysteroscopy Combined with Laparoscopy in Repairing 48 Patients with Uterine Scar Defect after Cesarean Section [J]. Clinical Medical Engineering, 2014, 021(012): 1573-1574.

[11] Wang Weifan. (2005). The Relationship between Liver and Reproduction [J]. Henan Traditional Chinese Medicine, 2005, 25(11): 9-11.

[12] Liu Shuhui, Yao Meiyu. (2010). “Three-step Method” for Treating Irregular Menstruation of Kidney Deficiency Type [J]. Jilin Traditional Chinese Medicine, 2010, 30(1): 26-27.

[13] Tian Sisheng. (2006). Zhu Danxi Medicine [M]. Beijing: China Traditional Chinese Medicine Press, 2006: 20.

[14] Yousefi Ghale-Salimi M., Eidi M., Ghaemi N., et al. (2018). Inhibitory effects of taraxasterol and aqueous extract of Taraxacum officinale on calci-um oxalate crystallization: in vitro study [J]. Ren Fail, 2018, 40(1): 298-305.

[15] Ben Khalifa B., Naouar S., Gazzah W., et al. (2016). Predictive factors of extra-corporeal shock wave lithotripsy success for urinary stones [J]. Tunis Med, 2016, 94(5): 397-400.

[16] Peng Wanda. (2019). Effects of Astragalus Polysaccharide on Insulin Resistance and Nrf2-ARE Pathway in Gestational Diabetic Rats [J]. Chinese Journal of Physicians, 2019, 21(10): 1507-1512, 1516.

[17] Wang Sihai, Lin Yixuan, Fang Chaohui, et al. (2020). Clinical study of endocrine and metabolic diseases treated with spleen [J]. Chinese Journal of Clinical Health, 2020, 23(1): 136-140.

[18] Xu Jian. (2010). On Kidney Deficiency and Menstrual Disease [J]. Chinese and Foreign Medical Research, $2010,8(26)$ : 172.

[19] Close the edge. Effect of Bushen Fu spleen Yangxue Decoction on Treating Menstrual Disease of Kidney Deficiency Type and Effect on Reproductive Hormone Level and Coagulation Status of Patients [J]. Chinese Medical Science, 2017(14).

[20] Zhong Xuan. (2017). Observation on serum endocrine hormone levels and autoimmunity antibodies in patients with endometriosis and infertility [J]. Shenzhen Integrated Chinese and Western Medicine Journal, 2017, 27(8): 12-13.

[21] Qin Qingshuang, Gao Meihua. (2016). Correlation between endometriosis and immune infertility [J]. Journal of Shandong Medical College, 2016, 38(1): 59-61.

[22] Xu Jing, Xia Qinchun. (2015). Clinical study on endocrine of women with endometriosis and infertility [J]. Health nutrition in China, 2015, 25(15): 49.

[23] Chen Yuanyuan, Lu Zhanbin. (2016). Effects of letrozole on endocrine hormones in endometriosis [J]. Chinese Science, 2016, 25(12): 65-67.

[24] Huang Wenjin, Yao Minglong, Ye Yunjin, et al. (2015). Application of Relevance Rules in Diagnosis of TCM Gynecology [J]. Hunan Journal of Traditional Chinese Medicine, 2015, (08): 148-149.

[25] Qu Qiyun. (2008). Treatment of endometriosis with soothing liver and regulating qi [J]. Henan Traditional Chinese Medicine, 2008, 28: 68-69.

[26] Wang Hong, Li Yuli, Sun Xiaoyu, et al. (2018). Diaphragm Zhuyu Decoction for Treatment of Endometriosis of Qi stagnation and Blood Stasis [J]. Journal of Chinese Medicine, 2018, 33(10): 188-192.

[27] Hou Jianling. (2008). Salvia miltiorrhiza Huoxue decoction for the treatment of endometriosis in 86 cases. Bright Chinese Medicine, 2008, 23(10): 1539.

[28] Fu Shuxiu, Lu Zhen, Gao Tianyu. (2007). Treatment of 46 Cases of Endometriosis with Buqi Quyu Prescription [J]. Chinese Medicine Clinical Journal, 2007, 19(3): 230-230.

[29] Yue Xiaoqiang. (2001). Historiography. Discussion on treatment of endometriosis with spleen [J]. Chinese Medicine, 2001, 12(3): 238-238.

[30] Wang Wenjuan, Yang Yanhong, Lu Zonglin. (2018). Effect of tonifying kidney and removing blood stasis in treating endometriosis infertility [J]. Chinese Folk Medicine Journal, 2018, 27(8): 83-84.

[31] Han Liyan, Bian Wenhui, Yang Bin, et al. (2012). Effect of Huazujiedu Bushen Quyu Prescription on FIH-1 $\alpha$ of Experimental Endometriosis Rats [J]. Jiangsu Traditional Chinese Medicine, 2012(09): 78-79. 